\title{
Probing the strange content of the proton via charm production in charged current deep inelastic scattering at HERA
}

\author{
Jae D. Nam ${ }^{* \dagger}$ \\ Temple University \\ E-mail: tuc12484@temple.edu
}

\begin{abstract}
This paper presents a preliminary result of measurement of charm production in charged current deep inelastic scattering (CCDIS) at HERA. The analysis was performed with all data sample taken during HERA II data-taking period, which corresponds to $e^{+} p$ and $e^{-} p$ collisions with a center-of-mass energy of $\sqrt{s}=318 \mathrm{GeV}$ with an integrated luminosity of $358 \mathrm{pb}^{-1}$. The charm cross sections for each lepton beam type are measured within a kinematic region $200<Q^{2}<$ $60000 \mathrm{GeV}^{2}, y<0.9, E_{T}^{j e t}>5 \mathrm{GeV}$ and $-2.5<\eta^{j e t}<2.5$, which can be extrapolated to the full phase space region. Charmed secondary interaction vertices were tagged by a lifetime-based method, which uses the decay lengths of secondary vertices to distinguish charmed events from light-flavoured events. Presented here are the preliminary results of visible charm cross sections in CCDIS as functions of $Q^{2}$.
\end{abstract}

ICHEP 2018, 39th International conference on High Energy Physics

4-11 July 2018

Seoul, Republic of Korea

* Speaker.

${ }^{\dagger}$ On behalf of ZEUS collaboration. 


\section{Motivation}

Among a number of measurements on the strange quark content of the proton are the oppositesign dimuon measurement at $\mathrm{CCFR} / \mathrm{NuTeV}$ and inclusive $W^{ \pm}$production measurement by ATLAS collaboration at LHC $[1,2]$. The strange quark content obtained from the two experiments differ by a factor of two albeit the measurements consider different regions in the kinematic phase space. This compels a revisit to this topic with a new approach at a different kinematic range.

HERA and ZEUS underwent a major luminosity and detector upgrade in the years 2000-2002 $[3,4]$. The data collected after this period is regarded as HERA II data. In order to fully exploit the improvements made during the upgrade period, this analysis uses all HERA II data. A lifetime based method was used to distinguish charmed jets from those containing only light quarks.

The data collected at ZEUS also provide a good testing ground for future physics studies with similar ep colliders, such as the Electron-Ion Collider (EIC) [5]. With the high-percision particle detection and final state identification of the EIC, many physics analyses performed at HERA, such as the present analysis, can be improved upon. This analysis seeks to test the feasibility of heavy flavour analyses at the EIC.

\section{Experiment}

This analysis uses all data sample taken at ZEUS during HERA II data-taking period. During this period, electrons and positrons of a beam energy $E_{e}=27.5 \mathrm{GeV}$ collided with protons of a beam energy $E_{p}=920 \mathrm{GeV}$ at a center-of-mass energy of $\sqrt{s}=318 \mathrm{GeV}$. The data were combined with respect to the charge of the incoming lepton, which corresponds to an integrated luminosity of $173 p b^{-1}$ for $e^{+} p$ collisions and an integrated luminosity of $185 p b^{-1}$ for $e^{-} p$ collisions. The kinematic reach of HERA allows for high $-Q^{2}$ measurements of charm production in CCDIS and the combination of $e^{+} p$ and $e^{-} p$ collisions enables a direct comparison between strange and antistrange quark content in the proton.

\section{Charm production in CCDIS}

The leading order charm production in CCDIS in $e^{ \pm} p$ collisions is a Quark-Parton model-like (QPM-like) process where a $W^{ \pm}$boson from the incoming lepton couples to a strange or antistrange quark in the proton sea. The same reaction is also possible via a down or anti-down quark replacing the strange flavour; however, this reaction is Cabbibo-suppressed.

Among the NLO charm production processes is the boson-gluon fusion. Here, the incoming $W$ boson couples to a $c \bar{c}$ pair produced from a gluon as $g W^{ \pm} \rightarrow c \bar{c} W^{ \pm} \rightarrow c \bar{s}(\bar{c} s)$. This process is sensitive to the gluon content in the proton. Thus, it compensates the higher QCD order as $x$ decreases and its contribution to the resulting charm production becomes comparable to the quark-initiated process. Both the QPM and BGF schemes need to be considered in order to correctly describe observable quantities. In the present paper, events with charm or anti-charm quarks involved in hard CC reaction either in the initial state (BGF) or final state (QPM) are collectively called electroweak charm events. 


\section{Analysis strategy}

The CC event selection, including ZEUS online trigger system, is discussed in detail elsewhere [7]. A characteristic of charged current events is that there is a large imbalance in the transverse momentum in the calorimeter. This is due to the neutrino in the final state, which is undetected and escapes the ZEUS detector. A selection cut was applied on the missing transverse momentum $p_{T, m i s s}>12 \mathrm{GeV}$ and measurements excluding the ones originated from the cells adjacent to the forward beam hole $p_{T, m i s s}^{\prime}>10 \mathrm{GeV}$. Another important criterion was kinematic selection requirement based on $Q^{2}$ and $y$. A kinematic cut was applied on $200<Q^{2}<60000 \mathrm{GeV}^{2}$ and $y<0.9$. This was to confine our data sample into a region with the optimal detector resolution. This also defines the kinematic phase space region of this analysis. About 4000 and 9000 events satisfied these selection criteria for $e^{+} p$ and $e^{-} p$ collision periods, respectively.

Charmed secondary interaction vertices in CCDIS events were then tagged by using the lifetime tagging method [8]. This method uses the longer decay lengths of heavy flavour hadrons due to their longer lifetimes in order to distinguish charmed hadrons from light flavour hadrons.

\section{Results}

The preliminary results of electroweak charm cross sections in CCDIS in $e^{ \pm} p$ collisions are provided in Figure 1 as functions of $Q^{2}$. The measurements were performed within the kinematic region of $200<Q^{2}<60000 \mathrm{GeV}^{2}, y<0.9, E_{T}^{j e t}>5 \mathrm{GeV}$ and $-2.5<\eta^{\text {jet }}<2.5$.

(a)

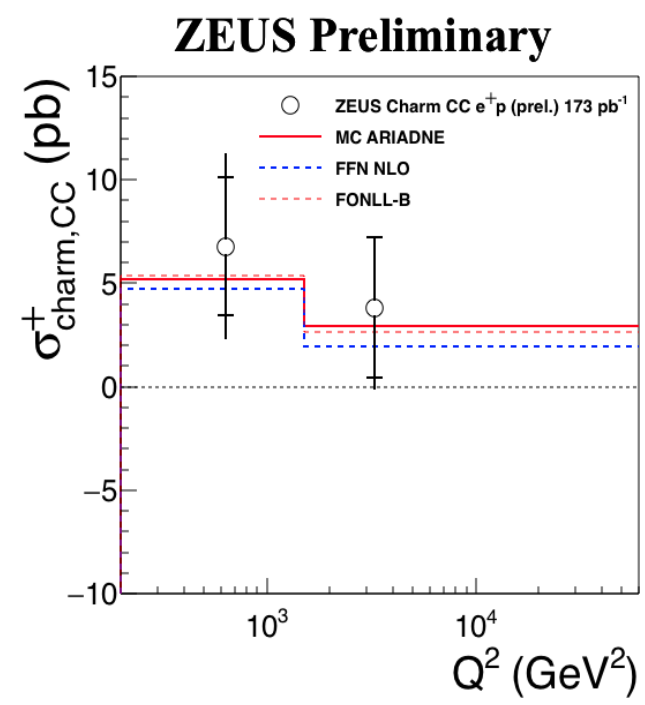

(b)

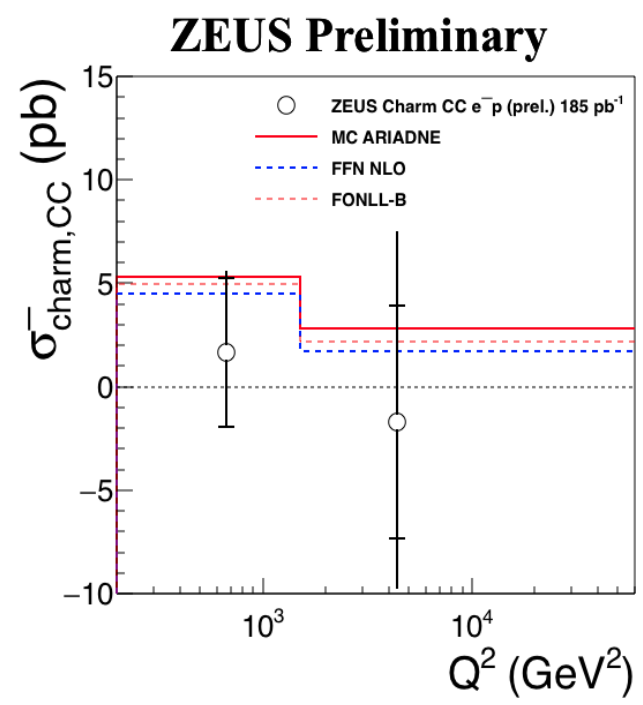

Figure 1: Total electroweak charm cross sections $\sigma_{\text {charm }, C C}$ in two bins of $Q^{2}$ in (a) $e^{+} p$ and (b) $e^{-} p$ collisions. The inner error bars show the statistical uncertainties and the full error bars show the total uncertainties. The solid red lines represent predictions obtained with MC. The dashed blue and dashed magenta lines represent predictions from FFN NLO and FONLL-B schemes, respectively. The theoretical and MC uncertainties are not included in the presented plots. 
The fixed-flavour number scheme (FFN) predictions were generated via OPENQCDRAD[10] for numerical computation with the ABMP 16.3 NLO PDF sets [11, 12]. The fixed-order nextto-leading log scheme (FONLL) predictions were generated by using the APFEL [13] code with NNPDF3.1. Both codes were interfaced in xFitter [14]. The theory predictions suggest that the quark-initiated process and BGF share about the same contribution to the resulting charm cross sections.

\section{Conclusions}

Measurements of the electroweak charm cross section in CCDIS have been performed for $e^{+} p$ and $e^{-} p$ collisions recorded at ZEUS within a kinematic region $200<Q^{2}<60000 \mathrm{GeV}^{2}$, $y<0.9, E_{T}^{j e t}>5 \mathrm{GeV}$ and $-2.5<\eta^{j e t}<2.5$. The measurements were performed separately for the incoming lepton type. Theoretical predictions were made within the FFN and FONLL schemes, which were found to describe the HERA II data well. Both suggested the contribution of BGF process to be about equal to that of the QPM process.

\section{References}

[1] A. O. Bazarko et al. [CCFR Collaboration], Z. Phys. C 65, 189 (1995)

[2] M. Aaboud et al. [ATLAS Collaboration], Eur. Phys. J. C 77, no. 6, 367 (2017)

[3] U. Schneekloth, DESY-HERA-98-05.

[4] A. Polini et al. [ZEUS Collaboration], Nucl. Instrum. Meth. A 581, 656 (2007)

[5] A. Accardi et al., Eur. Phys. J. A 52, no. 9, 268 (2016) doi:10.1140/epja/i2016-16268-9

[6] M. Tanabashi et al. [Particle Data Group], Phys. Rev. D 98, no. 3, 030001 (2018).

[7] H. Abramowicz et al. [ZEUS Collaboration], Eur. Phys. J. C 70, 945 (2010)

[8] F. D. Aaron et al. [H1 Collaboration], Eur. Phys. J. C 65, 89 (2010)

[9] S. D. Ellis and D. E. Soper, Phys. Rev. D 48, 3160 (1993)

[10] OPENQCD, http://www-zeuthen.desy.de/ alekhin/OPENQCDRAD

[11] S. Alekhin, J. Blumlein and S. Moch, Phys. Rev. D 86, 054009 (2012)

[12] I. Bierenbaum, J. Blumlein and S. Klein, Phys. Lett. B 672, 401 (2009)

[13] V. Bertone, S. Carrazza and J. Rojo, Comput. Phys. Commun. 185, 1647 (2014)

[14] S. Alekhin et al., Eur. Phys. J. C 75, no. 7, 304 (2015) 